Research Article

\title{
Improvement of the Angle of Arrival Measurement Accuracy for Indoor UWB Localization
}

\author{
N. Awarkeh, ${ }^{1}$ J.-C. Cousin ${ }^{1},{ }^{1}$ M. Muller, ${ }^{2}$ and N. Samama ${ }^{2}$ \\ ${ }^{1}$ LTCI, Telecom Paris, IP Paris, Paris, France \\ ${ }^{2}$ SAMOVAR, Telecom SudParis, IP Paris, Evry, France \\ Correspondence should be addressed to J.-C. Cousin; jean-christophe.cousin@telecom-paris.fr
}

Received 18 September 2019; Accepted 2 January 2020; Published 10 June 2020

Academic Editor: Vincenzo Stornelli

Copyright (C) 2020 N. Awarkeh et al. This is an open access article distributed under the Creative Commons Attribution License, which permits unrestricted use, distribution, and reproduction in any medium, provided the original work is properly cited.

\begin{abstract}
This paper shows that the accuracy of azimuth angle measurement for an interferometric localization system used to locate tags in its Line-of-Sight (LoS) can be improved by exploiting Impulse Radio-Ultra WideBand (IR-UWB) signals and without increasing the frequency bandwidth. This solution uses a Phase Correlation (PC) method, initially applied for Continuous Wave (CW) signals, adapted for Ultra WideBand (UWB) pulse signals. The obtained results are compared to those computed by a classical Energy Detection (ED) method where it becomes impossible to estimate azimuth angles for tag positions close to the orthogonal centered axis of the localization system baseline.
\end{abstract}

\section{Introduction}

In recent years, indoor localization has gained interest due to the vast range of services it can offer in many areas and applications. Many positioning technologies can be used within an indoor localization system depending on the intended accuracy, precision, cost, etc. The Global Navigation Satellite System (GNSS) is not accurate enough to provide specific services. Complementary systems have been developed to insure reliability and accuracy, even in hard conditions (multipaths, shadowing, ...). A solution is to use the time propagation of IR-UWB signal by exploiting wide frequency bandwidths and naturally increasing the accuracy.

Different approaches for UWB's estimated ToA are addressed in the literature. In [1], the performance of two different position estimation methods based on ToA estimation of the UWB signal for a set of receivers/sensors is investigated. The first one is based on the direct calculation method which gives the exact range (distance) between a tag and a sensor. The second one examines classical nonlinear optimization techniques, and more precisely the Davidon-FletcherPowell quasi-Newton algorithm. The optimal position is estimated by minimizing the summation of the quadratic errors for all the sensors. In [2], the problem of distance estimation in a dense multipath environment is studied by applying a technique that relies on the analysis of the power delay profile of the received UWB signal and the theory of segmentation of nonstationary processes. In [3], an algorithm combining match filtering and peak search techniques is presented to accurately determine the ToA of the received signal.

Most of the algorithms mentioned above are based on the ToA estimate of the strongest path. However, since the strongest path is not necessarily the first arrival path in dense multipath channels, their ToA accuracy is limited. Similarly, in some algorithms, prior knowledge of the received pulse shape also adds limitations to the ToA estimate. Therefore, with the change in shape of the different multipath components, it is more difficult to adapt exactly to the received pulse shape.

Due to these limitations, several solutions were proposed. In the first one, the detection is simplified by sending two pulses simultaneously so that the deformation affecting them will be the same. For instance, a two-step ToA estimation based on the time delayed sampling and correlation method for Transmitted-Reference UWB (TR-UWB) signals is presented in [4]. Another solution is based on energy detection, where energy is collected after certain analog front-end processing. In [5], the performance of three approaches based on 


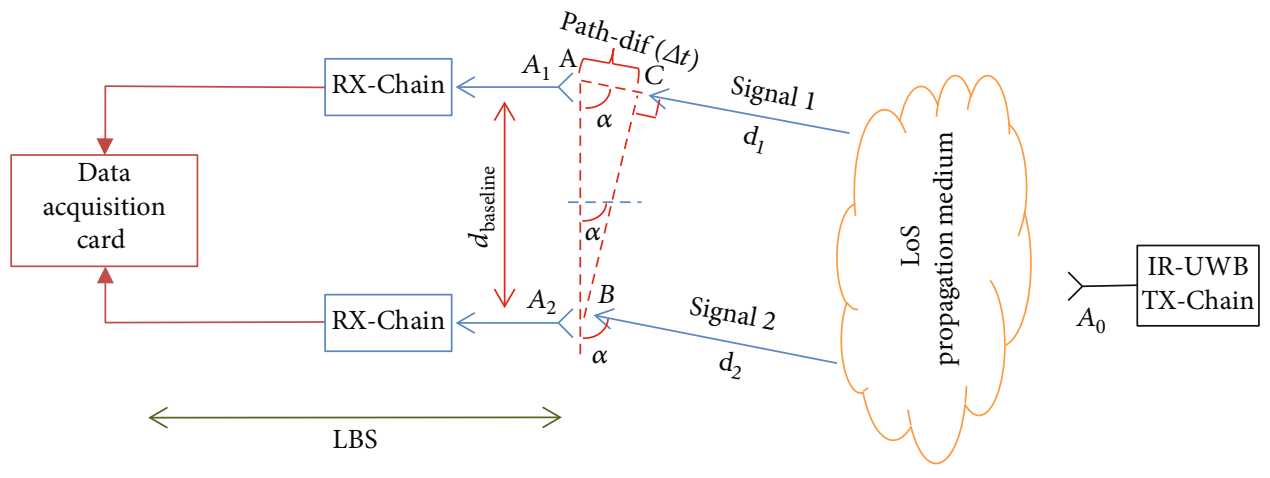

FIgURE 1: Architecture of the localization system.

ToA estimation techniques is analyzed for IR-UWB systems using sub-Nyquist sampling rates. These schemes, namely, Stored-Reference (SR), Transmitted-Reference (TR), and Energy Detection (ED), are used to collect the energy of the received signal. In [6], a two-step strategy based on SR and ED is applied to estimate the ToA, using a signal that corresponds to the IEEE 802.15.4a standard. The first step consists in finding the block with the highest energy. The second step consists in finding the chip where the first signal path is located, by correlating the received signal with a shifted version of the template signal. In [7], various ToA estimation algorithms for UWB systems are analyzed. These algorithms use a low sampling rate and three variants of the ED method, namely, Maximum Energy Selection (MES), Threshold Comparison (TC), and Maximum Energy Selection with Search-Back (MES-SB).

These algorithms do not require precise synchronization or pulse shapes but rather need a strong signal at the detector input. This requirement is crucial since the ToA estimation is seen as a problem of detecting the leading edge of the signal, which can be erroneous when the noise power is higher than the signal power.

Therefore, even if it is theoretically possible to obtain very accurate ranging, the practical limitations of the above algorithms prevent the localization system from achieving the desired high resolution. At the same time, a trade-off between the required accuracy, resolution, and complexity must be taken into account when developing a ToA estimation algorithm. To this end, we propose in this paper an alternative solution of low complexity based on Phase Correlation method adapted to IR-UWB signals, improving the accuracy and resolution of the azimuth angle measurement for an interferometric localization system. The main advantage of an interferometric method is that the position of a perso$\mathrm{n} /$ object in a room without obstacles can be obtained using only one fixed anchor instead of a well-known constellation.

\section{Localization System Design}

The 2D localization system, shown in Figure 1, consists of two main parts: the Localization Base Station (LBS) and an IR-UWB TX-Chain to be localized.

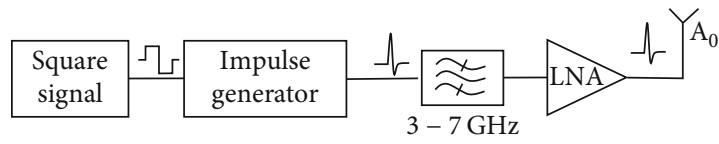

Figure 2: Architecture of the IR-UWB Tx-Chain.

2.1. Tx-Chain Architecture. The IR-UWB Tx-Chain architecture is depicted in Figure 2.

A square signal generator drives an impulse generator. In order to optimize the useful energy of the pulse in the bandwidth of $A_{0}$, the UWB pulse has been filtered by a band-pass filter (3-7 GHz) and next amplified by a LNA.

The obtained pulses at the output of the impulse generator, with a duration $T$ equal to $2 \mathrm{~ns}$, and at the input of the antenna $A_{0}$, with a duration $T^{\prime}>T$, are shown in Figures 3(a) and 3(b), respectively. $T^{\prime}$ is longer than $T$, and this is due to the distortions mainly caused by the group delay of the band-pass filter and the amplifier.

2.2. $R x$-Chain Architecture. The LBS architecture comprises two $\mathrm{RF}$ receiver chains ( $\mathrm{Rx}$-Chain), each connected to its own antenna, $A_{1}$ and $A_{2}$, respectively. These two antennas are placed on the same axis forming the baseline $\left(d_{\text {baseline }}\right)$. The two Rx-Chains are identical and shown in Figure 4.

The signal coming from the IR-UWB Tx-Chain is received by the two $\mathrm{Rx}$-Chain antennas $\left(A_{1}\right.$ and $\left.A_{2}\right)$ of the LBS. The received signals are amplified by a wideband LNA and then filtered by a band-pass filter (3-7 GHz). The acquisition system is an Agilent (Keysight) Infinium MSO 9254A acquiring simultaneously the two signals at the output of the band-pass filters.

\section{Azimuth Angle $\alpha$ Computation and Limitations}

The separation of the two receiving antennas $A_{1}$ and $A_{2}$ by $d_{\text {baseline }}$ creates a path difference (Path-dif) between two independent incoming signals (Signal 1 and Signal 2) which, in turn, gives the time difference $\Delta t=T_{2}-T_{1}$.

Path-dif $=c . \Delta t$ 


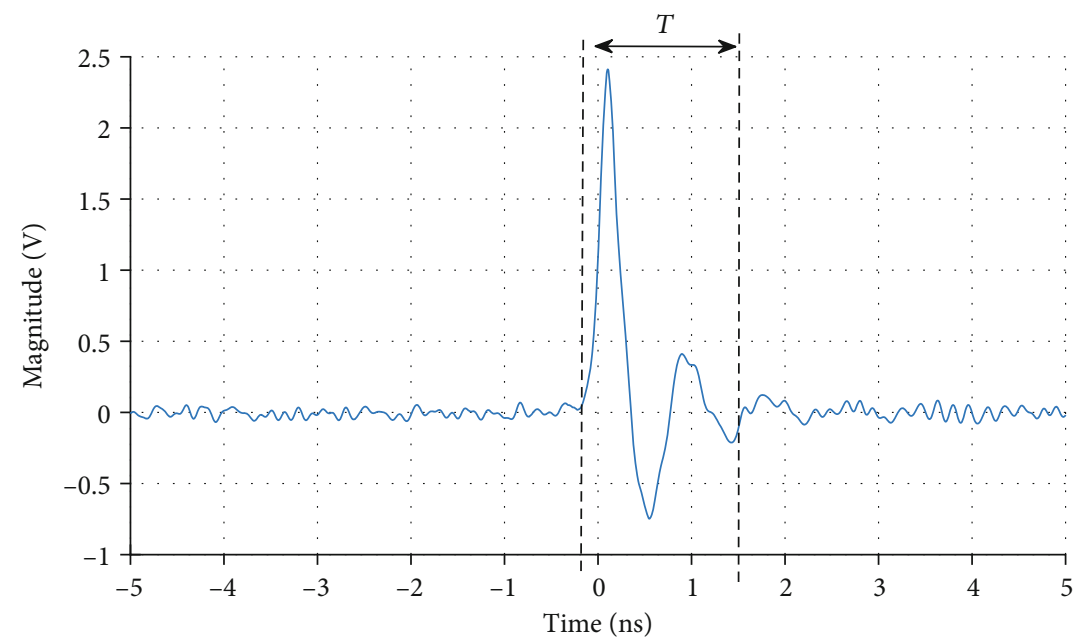

(a) The pulse at the impulse generator output

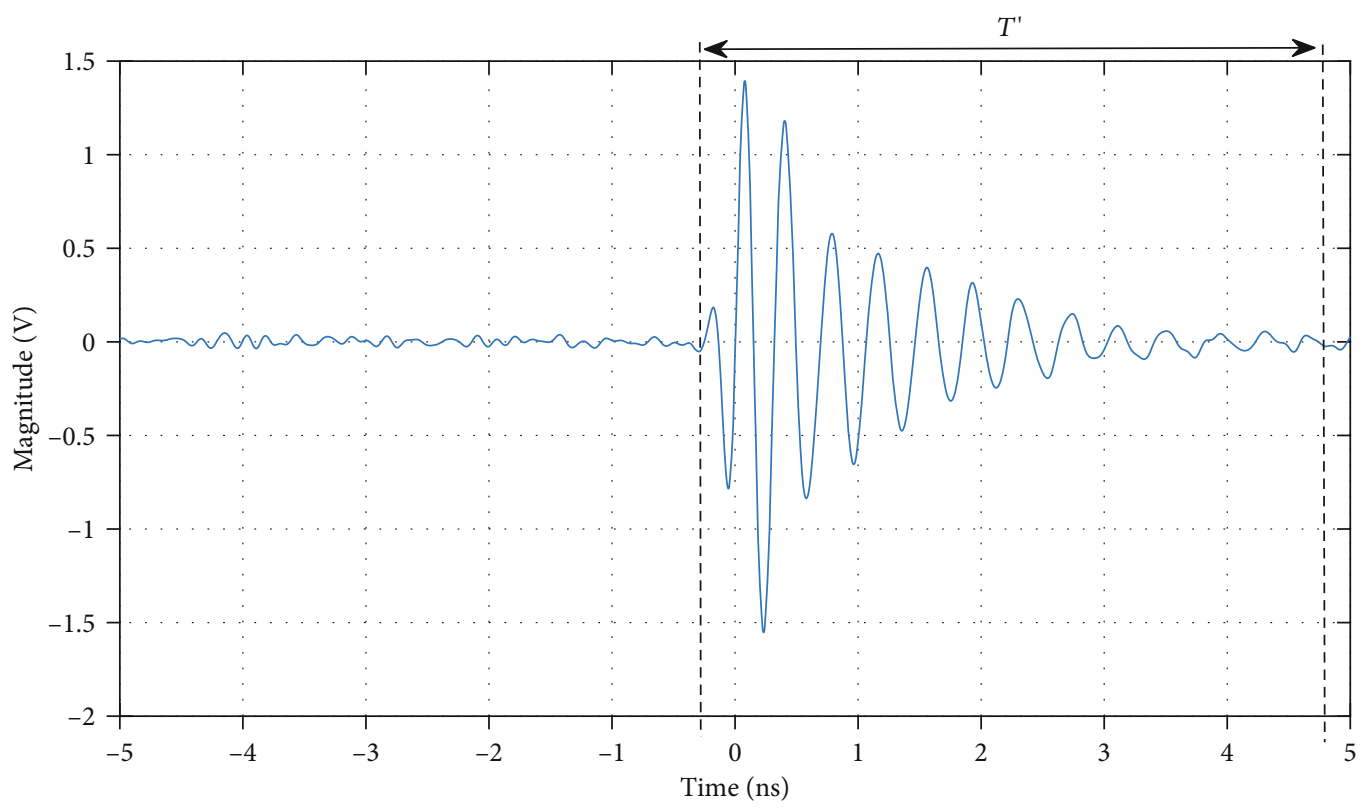

(b) The pulse at the antenna $A_{0}$ input

FIgURE 3: Shapes of the generated IR-UWB signal.

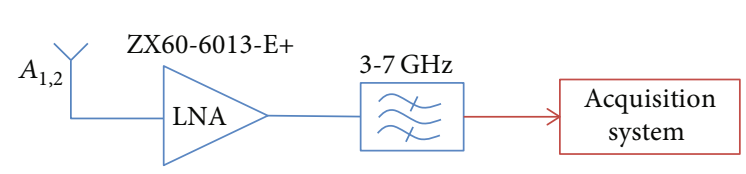

Figure 4: Architecture of the Rx-Chains.

On the other hand, for Signal 1 and Signal 2 being two plane waves, the Path-dif between these two parallel signals at an angle $\alpha$ with the baseline is given by the base length (AC) of the right-angle triangle $\mathrm{ABC}$. This assumption is only valid for a plane wave if the parallelism condition between the distances $d_{1}$ and $d_{2}$ is satisfied. Therefore, to fulfill this condition, we assume that $d_{1}$ and $d_{2}$ are large enough compared to $d_{\text {baseline }}[8]$.

$$
\text { Path-dif }=d_{\text {baseline }} \cdot \cos \alpha \text {. }
$$

The azimuth angle $\alpha$ measurement is computed as below [9]:

$$
\alpha=\cos ^{-1}\left[\frac{c\left(T_{2}-T_{1}\right)}{d_{\text {baseline }}}\right],
$$

where $T_{1}$ and $T_{2}$ are the time of flight of the signal over $d_{1}$ and $d_{2}$ and $c$ is the speed of light.

The pulse signal used for UWB radar applications generally covers the $3-5 \mathrm{GHz}$ bandwidth, called $\Delta_{f}$, which is the lower part of the UWB spectrum [10]. In general, the smallest Path-dif that the system can detect is equal to $c / 2 \Delta_{f}$. In our case, for a frequency bandwidth of $2 \mathrm{GHz}$, the Path-dif is 


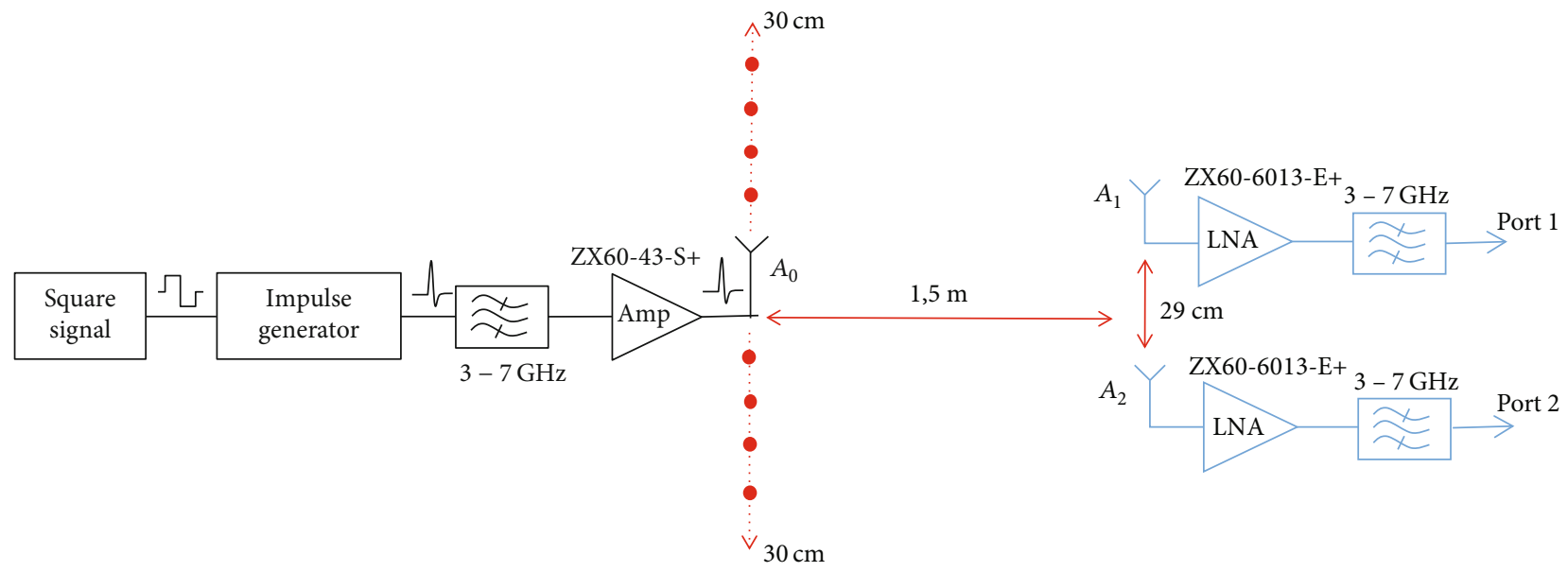

Figure 5: Test bench to measure the azimuth angle $\alpha$.

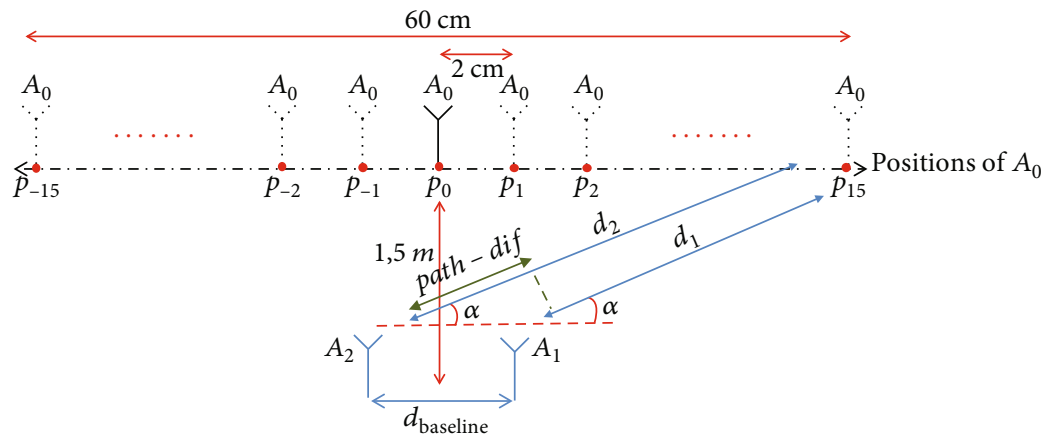

Figure 6: Possible positions of $A_{0}$.

$15 \mathrm{~cm}$ and the time difference $\left|T_{2}-T_{1}\right|$ is $0.5 \mathrm{~ns}$. So, using a $d_{\text {baseline }}=29 \mathrm{~cm}$, it becomes impossible to estimate the azimuth angle $\alpha$ in a range of $\left[60^{\circ}-120^{\circ}\right]$ due to a Path-dif less than $15 \mathrm{~cm}$.

To improve the accuracy of the azimuth angle measurement without increasing the frequency bandwidth, many solutions are proposed, such as [11] which exploits the phase difference of a CW signal. With a $d_{\text {baseline }}=29 \mathrm{~cm}$ and a CW signal at $2.45 \mathrm{GHz}$, the azimuth angle uncertainty range is equal to $+/-2^{\circ}$. The major drawback of these solutions is that the accuracy is affected by the multipath channel environment even in LoS situation.

An alternative solution to improve this accuracy is to exploit the distortion of the generated UWB pulse signal by the LBS (Tx-Chain and/or Rx-Chains) and the antennas instead of relying on CW signal. This method will be detailed in the next section.

\section{Accuracy Improvement for the Path-dif/Azimuth Angle Estimation}

The complete setup, shown in Figure 5, is composed by one $\mathrm{RF}$ transmitter chain linked to a circular polarized antenna $A_{0}$ and two RF receiver chains connected to two UWB horn antennas $A_{1}$ and $A_{2}$. The distance between $A_{1}$ and $A_{2}$ is denoted by $d_{\text {baseline }}$.

To improve the resolution of the path difference measurement between Signal 1 and Signal 2, the antenna $A_{0}$ can take 31 different positions $p_{i}$ with $i \in\{-15, \cdots, 15\}$, as shown in Figure 6. At the initial position $p_{0}$, the antenna $A_{0}$ is placed on an orthogonal axis at $1.5 \mathrm{~m}$ from the center of $d_{\text {baseline}}$, here equal to $29 \mathrm{~cm}$. The choice of $d_{\text {baseline }}$ equal to $29 \mathrm{~cm}$ is determined by the trade-off between the available equipment, the size of the receiving antennas $A_{1}$ and $A_{2}$, and the minimum value to insure a theoretical azimuth angle step measurement better than $1^{\circ}$. To validate the angular resolution improvement obtained with the PC method, $A_{0}$ can occupy several positions on a straight line parallel to the baseline, by shifting it $2 \mathrm{~cm}$ on each side of its initial position $p_{0}$ in the range $(-30 \mathrm{~cm} ; 30 \mathrm{~cm})$. The distance between $A_{0}$ and $A_{1}$ (resp., $A_{2}$ ) is denoted $d_{1}$ (resp., $d_{2}$ ). The path difference between the two received signals, Signal 1 and Signal 2, denoted Path-diff $=d_{1}-d_{2}$ is expressed in terms of the difference between their arrival times $T_{1}$ and $T_{2}$ at antennas $A_{1}$ and $A_{2}$, respectively. For this configuration, the maximum actual path difference corresponding to the positions $p_{-15}$ and $p_{15}$ is equal to $5.7 \mathrm{~cm}$.

In Figure 7, the recorded signals for the initial position $p_{0}$ are presented. 

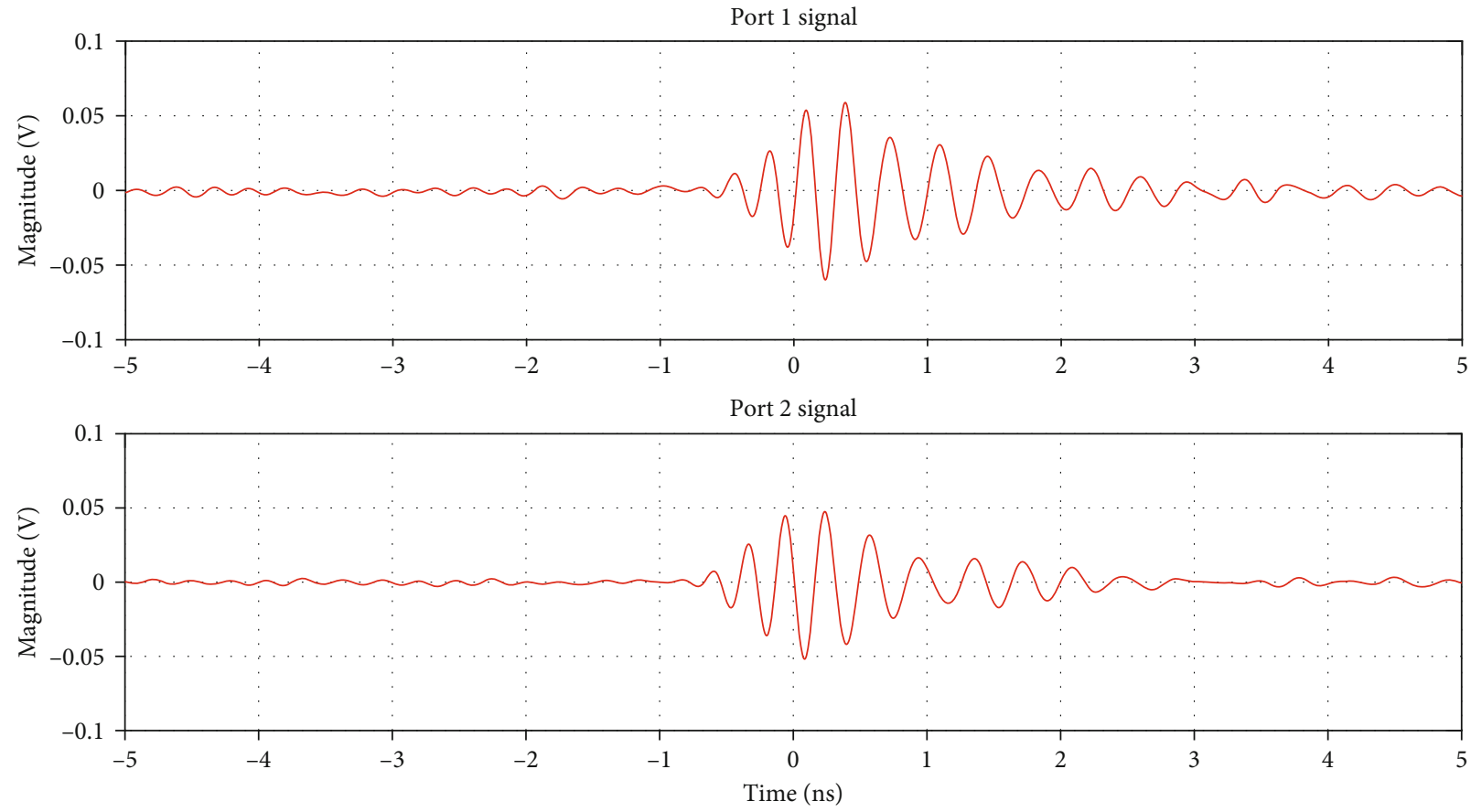

Figure 7: Recorded signals at Ports 1 and 2 for the initial position of $A_{0}$.

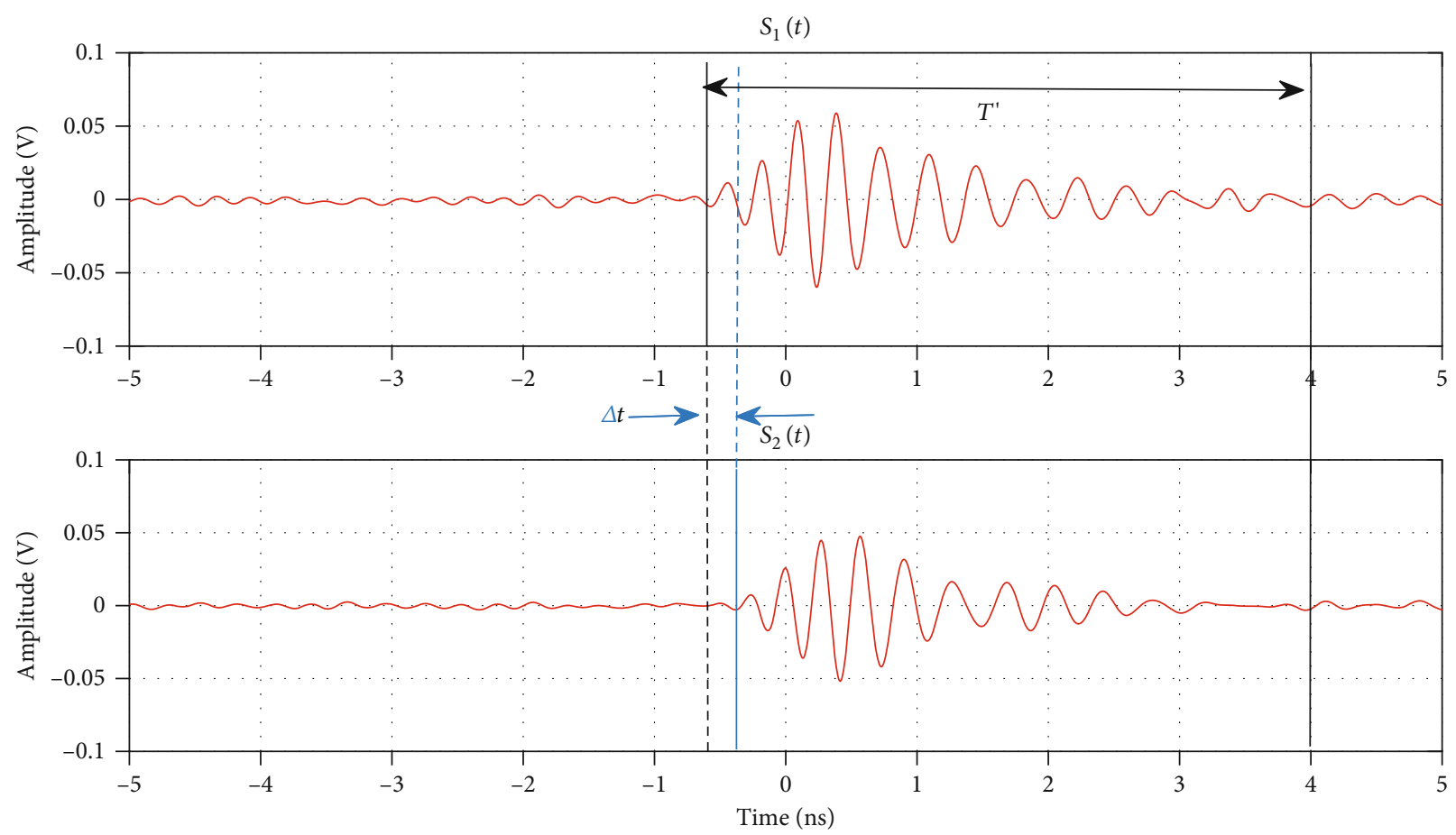

Figure 8: The $T^{\prime}, \Delta t$ parameter definition.

The two signals are distorted in the same way by the Tx and $\mathrm{Rx}$ components and the antennas. For these pulses, the signals $S_{1}(t)$ and $S_{2}(t)$, at Port 1 and Port 2, respectively, can be described by a sine carrier modulated by a Gaussian pulse [12] as

$$
\begin{aligned}
& S_{1}(t)=A \cos \left(2 \pi f_{0} t\right) e^{-\left(t / T^{\prime}\right)^{2},} \\
& S_{2}(t)=A \cos \left(2 \pi f_{0}(t-\Delta t)\right) e^{-\left(t-\Delta t / T^{\prime}\right)^{2}},
\end{aligned}
$$




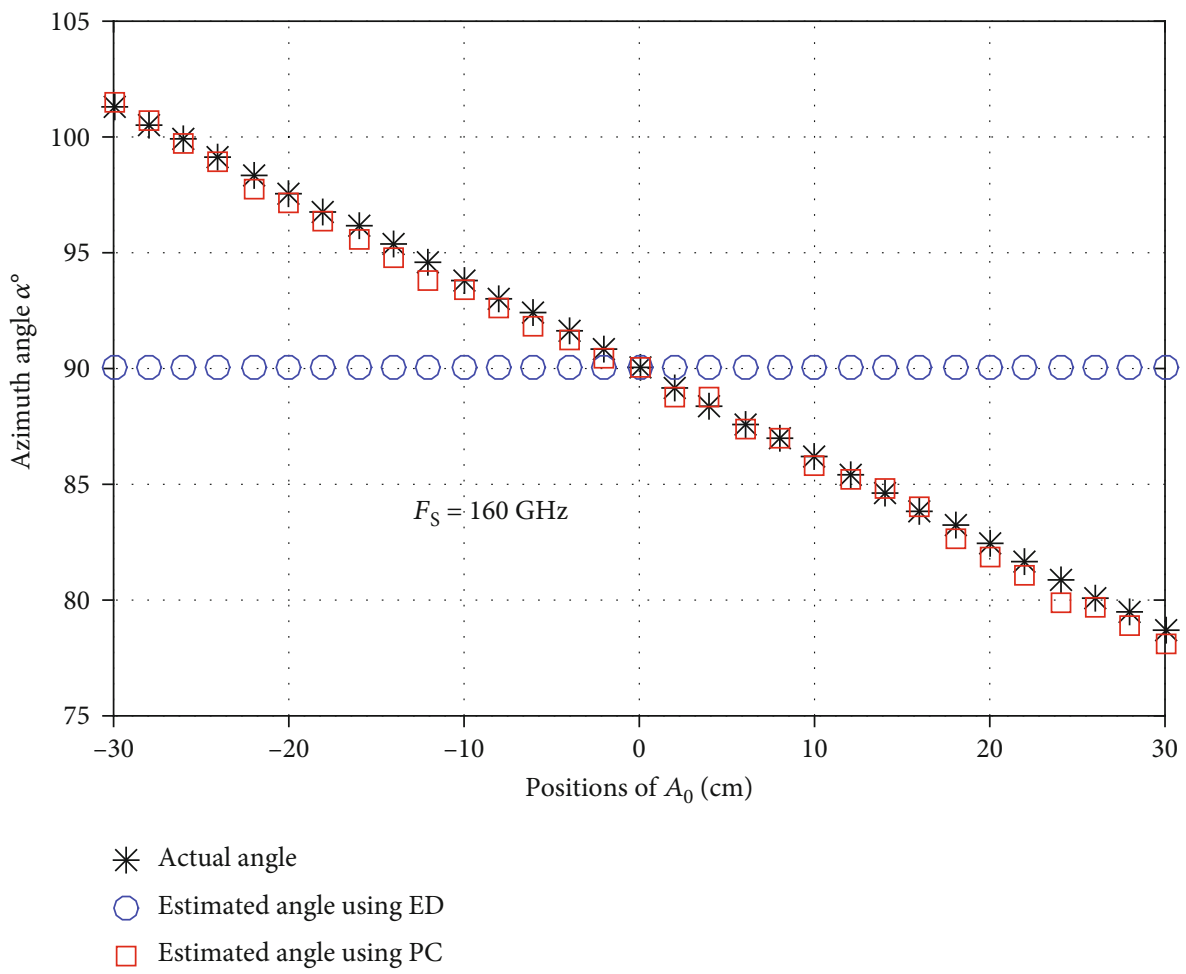

(a) Sampling frequency $F_{s}=160 \mathrm{GHz}$ (oversampling mode)

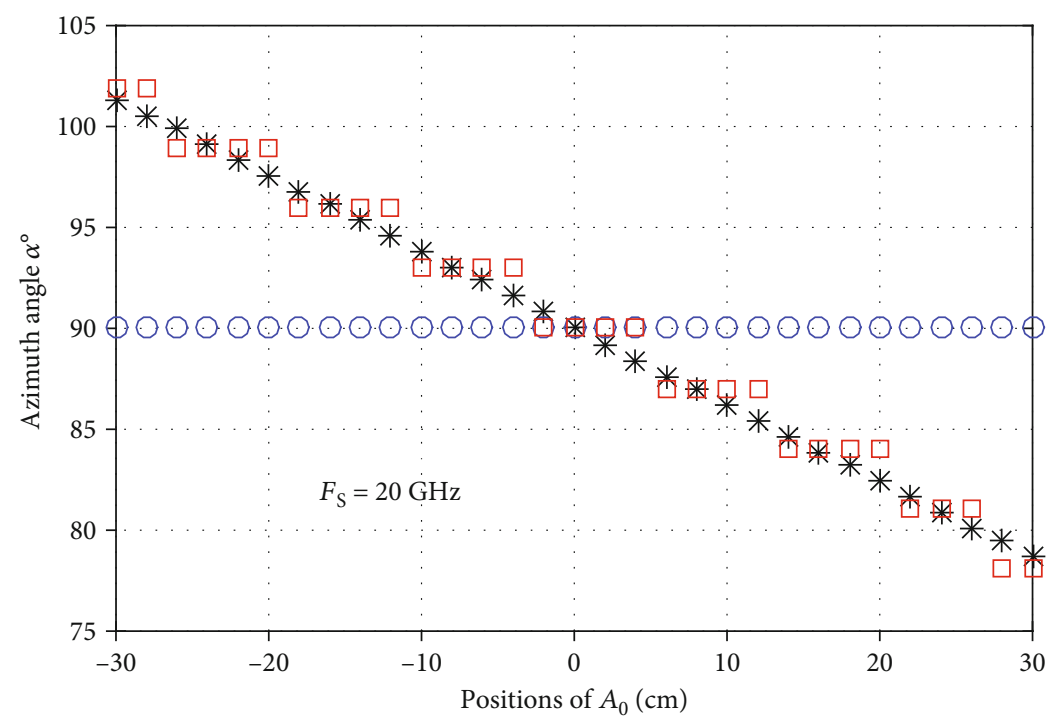

* Actual angle

Estimated angle using ED

Estimated angle using PC

(b) Sampling frequency $F_{s}=20 \mathrm{GHz}$

Figure 9: Azimuth angle estimation Vs Positions of $A_{0}$.

where $A$ is the signal magnitude, $f_{0}$ is the signal carrier frequency, $\Delta t=T_{2}-T_{1}$ is the delay for the Path-dif, and $T^{\prime}$ is the distorted pulse duration.

With an azimuth angle close to $90^{\circ}$, the Path-dif to measure is too small. In addition, the duration of the received pulse is much longer than the time difference $\left(T^{\prime} \gg \Delta t\right)$ when the path difference is very small (as shown in Figure 8).

The path difference between two received signals is estimated through the corresponding time difference $\Delta t$, obtained by correlating these two signals on the two receiving chains. 
By applying the cross-correlation function $C_{S_{1} S_{2}}(\Delta t)$ to the two received signals $S_{1}(t)$ and $S_{2}(t)$, we obtain

$$
C_{S_{1} S_{2}}(\Delta t)=\frac{A^{2}}{T} \int_{0}^{T} \underbrace{\cos \left(2 \pi f_{0} t\right) \cos \left(2 \pi f_{0}(t-\Delta t)\right)}_{B(t)} \underbrace{e^{-\left(\frac{t}{T^{\prime}}\right)^{2}} e^{-\left(\frac{t \Delta t}{T^{\prime}}\right)^{2}} d t . ~}_{C(t)}
$$

The duration analysis window $T$ is equal to the initial pulse width (Figure 3(a)), here 2 ns.

For a small path difference and $T, \Delta t \ll T^{\prime}$, the coefficients $B(t)$ and $C(t)$ can be written as

$$
\begin{aligned}
& B(t)=\cos \left(2 \pi f_{0} \Delta t\right) \frac{1+\cos \left(4 \pi f_{0} t\right)}{2}+\sin \left(2 \pi f_{0} \Delta t\right) \frac{\sin \left(4 \pi f_{0} t\right)}{2}, \\
& C(t)=e^{-2\left(t^{2} / T^{\prime 2}\right)-\left(\Delta t^{2} / T^{\prime 2}\right)+\left(2 \Delta t . t / T^{\prime 2}\right)} \approx 1 .
\end{aligned}
$$

So, the cross-correlation function $C_{S_{1} S_{2}}(\Delta t)$ becomes

$$
C_{S_{1} S_{2}}(\Delta t) \approx \frac{A^{2}}{T} \int_{0}^{T} B(t) d t \approx \frac{A^{2}}{2} \cos \left(2 \pi f_{0} \Delta t\right)
$$

which is considered as the Phase Correlation (PC) method directly related to the azimuth $\alpha$ through $\Delta t=T_{2}-T_{1}$ according to Equation (3).

\section{Experimental Results}

As proof of concept, we compare for each position of $A_{0}$ the results of the azimuth angle determination obtained by the conventional ED method [13] to the PC method with the configuration described in Figure 5. The ED method starts by partitioning the received signal, over the time, by blocks. Next, to estimate the ToA of the received signal, the block carrying the maximum energy is searched. So, with this method, the resolution is led by the size of blocks, here equal to $1 \mathrm{~ns}$, where the minimum size is fixed by the minimum energy able to be detected.

Therefore, the minimum path difference that the ED method can detect is $30 \mathrm{~cm}$. Thus, it is not able to detect a path difference in the order of $5.7 \mathrm{~cm}$ which corresponds to the maximum Path-dif obtained by this configuration (at $p_{-15}$ and $p_{15}$ ). On the other hand, the minimum path difference that the PC method can detect is determined by the sampling frequency, and therefore, it is able to detect a path difference of $1.5 \mathrm{~cm}$ when $F_{s}=20 \mathrm{GHz}$ and $0.2 \mathrm{~cm}$ when $F_{s}=160 \mathrm{GHz}$. This is directly reflected on the azimuth angle estimation since the path difference and the azimuth angle are related according to Equation (2). Note that, $F_{s}=160 \mathrm{GHz}$ is used only to show the theoretical limit of the method (ideal case).

In Figure 9, we present the azimuth angle versus the different positions of $A_{0}$ for $F_{s}$ equal to $160 \mathrm{GHz}$ (a) and $20 \mathrm{GHz}$ (b). The actual angle is compared to the ED and PC method estimation. We show that it is impossible to determine the azimuth angle $\alpha$ for short Path-dif with the ED method because the effective frequency bandwidth is reduced due to the time distortion of the pulse.

This becomes possible using the PC method in which the error is driven by the sampling frequency $F_{s}$. For instance, the error is less than $1^{\circ}$ for $F_{s}=160 \mathrm{GHz}$ (oversampling mode) and reaches $2^{\circ}$ for $F_{s}=20 \mathrm{GHz}$.

\section{Conclusion}

In this paper, we have shown that the measurement accuracy of the azimuth angle by a 2D interferometric indoor localization system is strongly improved. By jointly exploiting an IR-UWB signal, the distortions of this signal, and a Phase Correlation method, the improvement achieves an error of $1^{\circ}$ without increasing the frequency bandwidth even for angles producing very small Path-dif. This method can be extended to measure the elevation angle by adding a second $\mathrm{Rx}$-Chain architecture on an orthogonal axis to the first one.

\section{Data Availability}

The data used to support the findings of this study are available from the corresponding author upon request.

\section{Conflicts of Interest}

The authors declare that there is no conflict of interest regarding the publication of this paper.

\section{Acknowledgments}

This work was supported by the Institut Mines Telecom ("Futur et ruptures" grant).

\section{References}

[1] K. Yu and I. Oppermann, "Performance of UWB position estimation based on time-of-arrival measurements," in 2004 International Workshop on Ultra Wideband Systems Joint with Conference on Ultra Wideband Systems and Technologies. Joint UWBST \& IWUWBS 2004 (IEEE Cat. No.04EX812), pp. 400404, Kyoto, Japan, 2004.

[2] C. Mazzucco, U. Spagnolini, and G. Mulas, "A ranging technique for UWB indoor channel based on power delay profile analysis," in 2004 IEEE 59th Vehicular Technology Conference. VTC 2004-Spring (IEEE Cat. No.04CH37514), pp. 2595-2599, Milan, 2004.

[3] Z. N. Low, J. H. Cheong, C. L. Law, W. T. Ng, and Y. J. Lee, "Pulse detection algorithm for line-of-sight (LoS) UWB ranging applications," IEEE Antennas and Wireless Propagation Letters, vol. 4, pp. 63-67, 2005.

[4] J. A. Pardinas-Mir, R. Lamberti, M. Muller, and C. Gimenes, "An experimental approach to a low-complexity two-step TOA measurement for TR-UWB signals," in 2012 IEEE International Conference on Communications (ICC), pp. 45044508, Ottawa, ON, Canada, 2012.

[5] I. Guvenc, Z. Sahinoglu, and P. V. Orlik, "ToA estimation for IR-UWB systems with different transceiver types," IEEE Transactions on Microwave Theory and Techniques, vol. 54, no. 4, pp. 1876-1886, 2006. 
[6] S. Gezici, Z. Sahinoglu, A. F. Molisch, H. Kobayashi, and H. V. Poor, "A two-step time of arrival estimation algorithm for impulse radio UltraWideband systems," in 2005 13th European Signal Processing Conference, pp. 4-8, Antalya, Turkey, 2005.

[7] I. Guvenc and Z. Sahinoglu, "Threshold-based TOA estimation for impulse radio UWB systems," in 2005 IEEE International Conference on Ultra-Wideband, pp. 420-425, Zurich, Switzerland, 2005.

[8] R. Kumar, B. Huyart, J. C. Cousin, and K. Mabrouk, "2D measurement using interferometry and FMCW multistatic radar system for indoor localization," in European Radar Conference, pp. 17-20, Nuremberg, Germany, 2013.

[9] N. Awarkeh, J. C. Cousin, M. Muller, and N. Samama, "Improvement of the angle measurement accuracy for indoor UWB localization," in 2018 15th European Radar Conference (EuRAD), pp. 285-288, Madrid, Spain, 2018.

[10] T. W. Barrett, "History of ultra wideband communications and radar: part I, UWB communications," Microwave Journal-Technical Features, vol. 44, 2001.

[11] A. Benlarbi-Delai, J. C. Cousin, R. Ringot, A. Mamouni, and Y. Leroy, "Microwave short baseline interferometers for localization systems," IEEE Transactions on Instrumentation and Measurement, vol. 50, no. 1, pp. 32-39, 2001.

[12] D. D. Wentzloff and A. P. Chandrakasan, "Gaussian pulse generators for subbanded ultra-wideband transmitters," IEEE Transactions on Microwave Theory and Techniques, vol. 54, no. 4, pp. 1647-1655, 2006.

[13] I. Guvenc and Z. Sahinoglu, "Threshold selection for UWB TOA estimation based on kurtosis analysis," IEEE Communications Letters, vol. 9, no. 12, pp. 1025-1027, 2005. 